\title{
Emerging Role of Gut Microbiota beyond Infection
}

\section{Ibrahim Masoodi}

How to cite this article: Masoodi I. Emerging Role of Gut Microbiota beyond Infection. J Gastrointest Infect 2018;8(1):1-2

\section{Source of support: Nil}

\section{Conflict of interest: None}

Microbial assemblages colonizing the gastrointestinal (GI) tract are collectively referred to as the intestinal "microflora" or "microbiota." Members of this gut microbiota play a key role in maintaining human health by contributing to immune system maturation ${ }^{1}$ providing nutritional advantages to the host and promoting GI mucosal membrane integrity and function. ${ }^{2}$

The bacterial population of human intestinal microbiota comprises up to 2,000 different species. ${ }^{3}$ Human intestinal microbiota diversity has been thoroughly characterized by sequencing 13,335 prokaryotic ribosomal RNA genes from the stool and mucosal sites of three individuals ${ }^{4}$ and, more recently, by sequencing 18,348 fecal bacterial $16 \mathrm{~S}$ rRNA genes collected from 14 unrelated adults. ${ }^{5}$ These studies revealed the presence of up to 400 bacterial phylotypes, among which $80 \%$ represented rRNA sequences from as-yet-uncultured or novel bacteria. The composition of gut microbiota between individuals often shares similar high-level phylogenetic relationships ${ }^{6}$ although each individual harbors specific gut microbiota at the species and/or strain level. ${ }^{7}$

The use of germ-free animals has clearly demonstrated the beneficial role of gut microbiota in maintaining homeostasis. The positive effect of the intestinal microbiota on the host is directly dependent on its qualitative and quantitative composition. An imbalance in gut microbiota has been associated with several chronic GI disorders, including irritable bowel syndrome (IBS), ${ }^{8}$ inflammatory bowel disease (IBD) and colorectal cancer (CRC). Emerging evidence suggests a possible role of gut microbiota in IBS pathogenesis. ${ }^{9}$ The evidence in support is the existence of a post-infectious variant of IBS. Further the beneficial effects conferred by specific antibiotics or probiotics in IBS also supports possible role of bacteria in

\footnotetext{
${ }^{1}$ Associate Professor

${ }^{1}$ Department of Gastroenterology, College of Medicine, Taif University, Kingdom of Saudi Arabia
}

Corresponding Author: Ibrahim Masoodi, Department of Gastroenterology, College of Medicine, Taif University, Kingdom of Saudi Arabia, e-mail: ibrahimmasoodi@yahoo.co.in one of the variants of IBS. ${ }^{10}$ According to many of these studies, the gut microbial community is significantly altered in IBS. However, whether such changes are causal, consequential, or merely the result of constipation and diarrhea remains unclear.

Numerous studies have revealed alterations in the gut microbiota composition of patients with IBD. Dickseverd et $\mathrm{al}^{11}$ recently characterized the gut microbiota of 10 monozygotic twin pairs with Crohn's disease (CD) and 8 healthy twin pairs. The microbial populations from the healthy twins were highly similar, while the populations differed between twins with CD. Decreased bacterial diversity was observed in the patients with $C D$ compared to the healthy individuals; this decrease was partially explained by changes in the Bacteroides community representatives. More specifically, the patients with CD had microbiota profiles that were characterized by decreased B. uniformis abundance but increased B. avotus and B. vulgatus abundance as compared to those of healthy individuals. ${ }^{11}$ Interestingly, Franck and collaborators also identified members of the Bacteroides genus as potential biomarkers predicting disease predisposition and severity. ${ }^{12}$

The gut microbiota composition seems to modulate the risk of CRC development. In fact, the presence of B. vulgatus, B. stercoris, and Clostridia spp. has been directly linked to a high risk of CRC in humans. ${ }^{8}$

The exact microbial interactions within the human gut are complicated and remain poorly understood. However, alterations in gut microbiota composition due to various environmental factors may act as a trigger for conditions such as IBS, IBD, or CRC, ${ }^{13}$ just as microbiota alterations in various tissues may increase an individual's susceptibility to other diseases. For example, microbial gene function alterations, such as an increase in methanogenesis pathway activity due to the presence of Methanobrevibacter, may predispose patients to certain intestinal diseases.

Further insight in these complex processes has been provided by metagenomic studies. Metagenomics is a molecular method of culture-independent microbiology, in which genetic material recovered directly from environmental samples is studied. It has emerged as one of the most robust sequence-driven approaches for studying the composition and the genetic potential of the mucosal gut microbiota. Metagenomics analysis has begun to demonstrate the breadth of the functional and metabolic 
potential of microbes. It has been used to demonstrate significant metabolic discrepancies between diseased and healthy individuals. Although the intestinal microbiota in individuals reflects great variations amongst people according to their age, geographic origin, state of heath, and variations in diet, it tends to remain stable over long periods. ${ }^{14}$

To conclude gut microbes play a very important role beyond infection, and metagenomics may help to understand pathogenesis, and treatment of various chronic GI disorders in near future.

\section{REFERENCES}

1. Abt MC, Artis D. The intestinal microbiota in health and disease: the influence of microbial products on immune cell homeostasis. Curr Opin Gastroenterol 2009; 25: 496-502 [PMID: 19770652 DOI: 10.1097/MOG.0b013e328331b6b4]

2. Barbara G, de Giorgio R, Stanghellini V, Cremon C, Salvioli B, Corinaldesi R. New pathophysiological mechanisms in irritable bowel syndrome. Aliment Pharmacol Ther 2004; 20 Suppl 2: 1-9 [PMID: 15335408 DOI: 10.1111/j.1365-2036.2004.02036.x]

3. Backhed F, Ley RE, Sonnenburg JL, Peterson DA, Gordon JI. Host-bacterial mutualism in the human intestine. Science 2005; 307: 1915-1920 [PMID: 15790844 DOI: 10.1126/ science.1104816]

4. Dicksved J, Halfvarson J, Rosenquist M, Jarnerot G, Tysk C, Apajalahti J, Engstrand L, Jansson JK. Molecular analysis of the gut microbiota of identical twins with Crohn's disease. ISME J 2008; 2: 716-727

5. Eckburg PB, Bik EM, Bernstein CN, Purdom E, Dethlefsen L, Sargent M, Gill SR, Nelson KE, Relman DA. Diversity of the human intestinal microbial flora. Science 2005; 308: 1635-1638 [PMID: 15831718 DOI: 10.1126/science.1110591]

6. Frank DN, St Amand AL, Feldman RA, Boedeker EC, Harpaz N, Pace NR. Molecular-phylogenetic characterization of microbial community imbalances in human inflammatory bowel diseases. Proc Natl Acad Sci U S A 2007; 104: 13780-13785

7. Ley RE, Backhed F, Turnbaugh P, Lozupone CA, Knight RD, Gordon JI. Obesity alters gut microbial ecology. Proc Natl Acad Sci U S A 2005; 102: 11070-11075 [PMID: 16033867 DOI: 10.1073/pnas.0504978102]

8. Ley RE, Turnbaugh PJ, Klein S, Gordon JI. Microbial ecology: human gut microbes associated with obesity. Nature 2006; 444: 1022-1023 [PMID: 17183309 DOI: 10.1038/4441022a]

9. Ley RE, Hamady M, Lozupone C, Turnbaugh P, Ramey RR, Bircher JS, Schlegel ML, Tucker TA, Schrenzel MD, Knight $\mathrm{R}$, Gordon JI. Evolution of mammals and their gut microbes. Science 2008; 320: 1647-1651 [PMID: 18497261 DOI: 10.1126/ science.1155725]

10. Moore WE, Moore LH. Intestinal floras of populations that have a high risk of colon cancer. Appl Environ Microbiol 1995; 61: 3202-3207 [PMID: 7574628]

11. Pimentel M, Park S, Mirocha J, Kane SV, Kong Y. The effect of a nonabsorbed oral antibiotic (rifaximin) on the symptoms of the irritable bowel syndrome: a randomized trial. Ann Intern Med 2006; 145: 557-563

12. Turnbaugh PJ, Backhed F, Fulton L, Gordon JI. Diet-induced obesity is linked to marked but reversible alterations in the mouse distal gut microbiome. Cell Host Microbe 2008; 3: 213-223

13. Xu J, Gordon JI. Honor thy symbionts. Proc Natl Acad Sci U S A 2003; 100: 10452-10459

14. Zou S, Fang L, Lee MH. Dysbiosis of gut microbiota in promoting the development of colorectal cancer. Gastroenterol Rep (Oxf). 2018 Feb;6(1):1-12. 